\title{
A comparison of video and autofluorescence bronchoscopy in patients at high risk of lung cancer
}

\author{
P.N. Chhajed, K. Shibuya, H. Hoshino, M. Chiyo, K. Yasufuku, \\ K. Hiroshima and T. Fujisawa
}

ABSTRACT: The aim of this study was to compare the diagnostic yield of flexible video bronchoscopy (FVB) and autofluorescence bronchoscopy (i.e. lung imaging fluorescence endoscopy (LIFE)) in 151 patients at a high risk of lung cancer and with moderate dysplasia or worse on sputum cytology mass screening.

Findings from FVB and LIFE were classified as either normal, abnormal or suspicious for cancer. Endobronchial biopsies (EBX) were obtained from abnormal or suspicious areas on FVB and/or LIFE, or randomly when FVB and LIFE were normal. Moderate dysplasia and worse were defined as pathologically positive.

Overall, 83 out of 343 (24\%) EBX were pathologically positive. The sensitivity of FVB was $72 \%$ and LIFE $96 \%$. Relative sensitivity of LIFE over FVB was 1.33. Specificities of FVB and LIFE were 53 and $23 \%$, respectively. The numbers of pathologically positive EBX from sites designated normal, abnormal or suspicious were: from FVB, 23 out of 162 (14\%), 37 out of 151 (25\%) and 23 out of $30(77 \%)$; from LIFE, three out of $69(4 \%), 44$ out of $212(21 \%)$ and 36 out of $62(58 \%)$. In normal or abnormal areas at FVB, there was a significant increase in the yield of EBX guided by abnormal and suspicious sites noted at LIFE.

In conclusion, endobronchial biopsies of suspicious findings from lung imaging fluorescence endoscopy and flexible video bronchoscopy have a good diagnostic yield. Lung imaging fluorescence endoscopy is more useful when flexible video bronchoscopy is either normal or abnormal.

KEYWORDS: Dysplasia, intra-epithelial neoplasia, lung cancer, lung imaging fluorescence endoscopy, video bronchoscopy

$\mathbf{T}$ he proportion of individuals with bronchial carcinoma in situ in whom invasive cancer will develop is likely to be $>40 \%$ [1]. Treatment of cervical dysplasia or carcinoma in situ has significantly reduced the incidence and mortality of invasive cervical cancer [2]. By coupling sensitive diagnostic tools used in the investigation of early lung cancer with new treatment modalities, such as chemoprevention and various endobronchial therapies, it is hoped that the traditionally poor prognosis for patients with lung cancer can be altered in the near future [1]. An important factor needed for the success of such programmes is the easy availability of diagnostic tools.

The effectiveness of autofluorescence bronchoscopy (e.g. lung imaging fluorescence endoscopy (LIFE)) as a guide for endobronchial biopsy has been established for the localisation of intraepithelial neoplasia [3, 4]. Endoscopic changes have been described using white light bronchoscopy that can be used to detect dysplasia and carcinoma in situ [5]. Several studies have described the benefit of autofluorescence bronchoscopy over white light bronchoscopy [3, 6 , 7]. In a multicentre study, LAM et al. [3] reported that the addition of autofluorescence bronchoscopy to white light bronchoscopy resulted in the detection of a significant number of early intra-epithelial neoplastic lesions that would otherwise have been missed. In these studies, white light bronchoscopy was carried out using a fibreoptic bronchoscope. The white light flexible video bronchoscope (FVB) has a miniature charge couple device built into its tip, which helps to deliver a clearer image. Furthermore, the images obtained with the FVB do not have the

\section{AFFILIATIONS}

Dept of Thoracic Surgery, Graduate School of Medicine, Chiba

University, Chiba, Japan.

\section{CORRESPONDENCE}

T. Fujisawa

Dept of Thoracic Surgery Graduate School of Medicine Chiba University

1-8-1 Inohana

Chuo-ku

Chiba 260-8670

Japan

Fax: 81432262172

E-mail: fujisawat@faculty.chiba-u.jp

Received:

January 312004

Accepted after revision:

January 272005 
graininess seen with images obtained from the conventional fibreoptic bronchoscope. To the current authors' knowledge, the utility of FVB and autofluorescence bronchoscopy, specifically LIFE, has not been compared previously in the investigation of individuals at a high risk of lung cancer (moderate dysplasia or worse on sputum cytology) to localise intra-epithelial dysplasia or neoplasia. Hence, this study was undertaken to correlate the findings observed with FVB and LIFE, and to assess their diagnostic yield in the investigation of individuals at a high risk of developing lung cancer.

\section{METHODS}

From September 1998-September 2002, 158 consecutive highrisk individuals, who underwent mass screening for lung cancer having sputum cytology findings of class C, D and E, as defined by the Japan Lung Cancer Society, were referred to the Dept of Thoracic Surgery, Chiba University, Chiba, Japan, for bronchoscopic investigation [8]. Criteria for cytological diagnosis by sputum cytology were: A) no histiocytes in sputum (insufficient specimen); B) normal, only normal epithelial cells, proliferative basal cells, mild dysplasia and ciliated columnar cells; C) moderate dysplasia and columnar cells with changes of nuclear size or strong staining; D) severe dysplasia or cells suspicious for malignancy; and E) cells of malignant tumour [4, 8]. All patients underwent chest radiography and computed tomography of the chest. Patients with peripheral lesions underwent transbronchial biopsy in addition to transbronchial needle aspiration cytology under fluoroscopy guidance as they had abnormal sputum cytology. None of the patients had previously received photosensitising agents, such as haematoporphyrin derivative, chemopreventative drugs, radiotherapy to the chest, or cytotoxic chemotherapy agents. Seven patients were excluded (four due to inadequate endobronchial biopsy specimens and three due to vocal cord malignancy). Patients with vocal cord malignancy were excluded from the analysis to maintain a uniform cohort, as none of the remaining 151 patients included in the study had a known extrapulmonary cancer.

All patients underwent FVB (BF-240; Olympus, Tokyo, Japan) followed by LIFE (Xillix LIFE; Xillix Technologies Corp., Richmond, BC, Canada) in the same session, which was applied using a fibreoptic bronchoscope (BF40; Olympus). Findings of FVB and LIFE were documented prospectively during the procedure and were not changed once the procedure was completed. FVB was performed under local anaesthesia and sedation (diazepam/midazolam). Visual findings at FVB were first recorded either as normal, abnormal or suspicious for high-grade dysplasia or cancer, as follows. Normal: areas without any visual abnormality; abnormal: areas with increased redness and hypervascularity, swelling or thickening of the bronchial mucosa, or focal thickening of a subcarina; suspicious for high-grade dysplasia or cancer: nodular or polypoid lesions or irregularity of the bronchial mucosa (fig. 1) [3, 6, 9]. FVB was followed by LIFE, and findings were also recorded as either normal, abnormal or suspicious [3]. With LIFE bronchoscopy, normal areas appear as green; areas with a definite brown or brownish-red colour (loss of green fluorescence) were labelled as suspicious for high-grade dysplasia or cancer; and areas that were slightly brown with ill-defined margins were labelled as abnormal.
Endobronchial biopsies (EBX) were obtained using 2-mm diameter fenestrated cup forceps (FB-19C-1; Olympus) from abnormal or suspicious areas at both FVB and/or LIFE. In patients with normal FVB and LIFE, EBX was obtained from a second-generation carina. Biopsy specimens were reported and classified as either: 1) normal; 2) inflammation/bronchitis; 3) hyperplasia; 4) squamous metaplasia; 5) mild dysplasia; 6) moderate dysplasia; 7) severe dysplasia; 8) carcinoma in situ; or 9) carcinoma $[4,10]$.

Histological findings from 1-5 were classified as negative and 6-9 as positive [3]. Relative sensitivity was calculated to evaluate the contribution of LIFE to FVB to detect intraepithelial dysplasia or neoplasia [3]. Relative sensitivity of $>1$ would indicate a significant improvement of LIFE over FVB. The Chi-squared test was used to assess the association between the findings of FVB and LIFE with the pathological diagnosis.

\section{RESULTS}

A total of 151 patients (142 males, nine females) with a mean age $67 \pm 8$ yrs (range $44-82$ yrs) underwent EBX from 343 sites. A history of smoking was present in 143 (95\%) individuals with a mean of $54 \pm 24$ pack-yrs (range 11-153). Sputum cytology classes C, D and E were present in 42 (28\%), 58 (39\%) and $50(33 \%)$ individuals, respectively. Clear interpretation of sputum cytology was not possible in one patient with haemoptysis. The pathological and bronchoscopic characteristics of the EBX are presented in table 1. Normal, abnormal and suspicious areas were observed during FVB at 162 (47\%), $151(44 \%)$ and 30 (9\%) biopsy sites, respectively; and LIFE at 69 $(20 \%), 212(62 \%)$ and $62(18 \%)$ biopsy sites, respectively (table 2). Abnormal or suspicious findings during FVB and/ or LIFE were present at 295 out of $343(86 \%)$ biopsy sites.

Of the 83 out of 343 (24\%) pathologically positive EBX, 81 out of $295(27 \%)$ were obtained from sites that had abnormal or suspicious findings at FVB or LIFE, and two out of $48(4 \%)$ were obtained from areas that were normal at both FVB and LIFE. The sensitivity of FVB was $72 \%$ (60 out of 83 ) and LIFE $96 \%$ (80 out of 83 ). The relative sensitivity of LIFE over FVB was 1.33 ( 80 over 60 ). The specificities of FVB and LIFE were $53 \%$ (139 out of 260 ) and $23 \%$ (66 out of 260 ), respectively. The positive and negative predictive values of FVB were 60 out of $181(33 \%)$ and 139 out of $162(86 \%)$, respectively; and for LIFE 80 out of $274(29 \%)$ and 66 out of 69 (96\%), respectively.

\section{Value of LIFE when biopsy sites are normal at FVB}

In areas that were visualised to be normal during FVB (162 biopsy sites), there was a significant increase in the yield of pathologically positive EBX guided by abnormal and suspicious lesions noted with LIFE (21 out of 114; 18\%) compared with the random biopsies obtained from sites with normal LIFE (two out of $48 ; 4 \%$; $<0.05$ ). Random biopsies obtained from areas with normal FVB and LIFE accounted for two out of $83(2 \%)$ of the total positive biopsies.

\section{Value of LIFE when biopsy sites are abnormal or suspicious at FVB}

In areas with abnormal FVB findings (151 biopsy sites), there was a significant increase in the yield of pathologically positive biopsies obtained from sites with abnormal and suspicious 

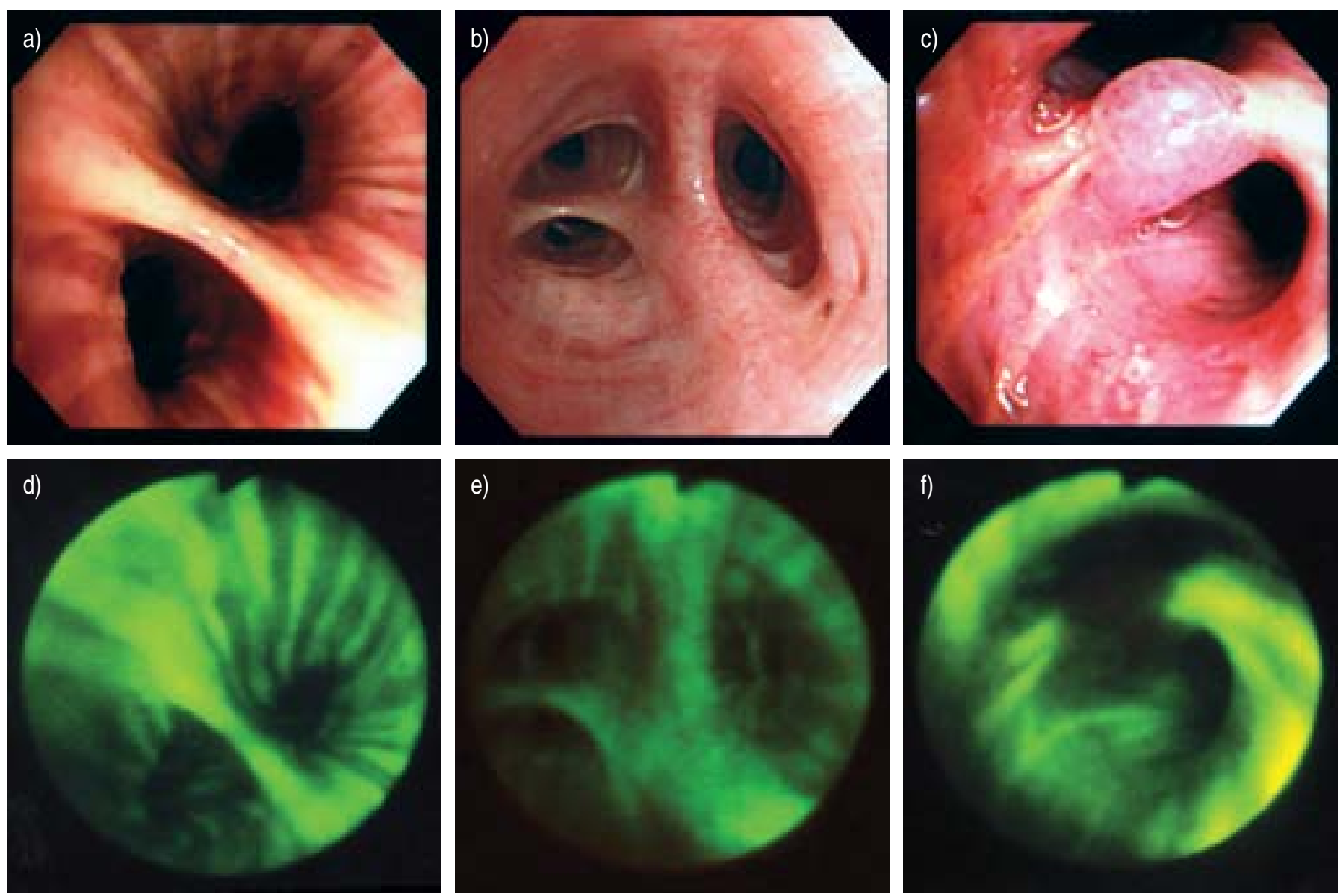

FIGURE 1. a) and d) show normal appearance with flexible video bronchoscopy (FVB) and abnormal lesion with lung imaging fluorescence endoscopy (LIFE), respectively, at the same site. b) and e) show abnormal appearance at FVB and suspicious lesion at LIFE, respectively, at the same site. c) and f) show suspicious lesions at both FVB and LIFE, respectively, at the same site.

LIFE (36 out of 130; $28 \%$ ) when compared with normal LIFE (one out of $21 ; 5 \% ; \mathrm{p}<0.05$ ). Biopsies obtained from areas with abnormal FVB and normal LIFE accounted for one out of 83
$(1 \%)$ of the total positive biopsies. All the 30 areas with suspicious findings on FVB also had suspicious changes on LIFE. All of the three lesions with carcinoma in situ were

TABLE 1 Pathological and bronchoscopic characteristics of 343 endobronchial biopsy sites

\begin{tabular}{|c|c|c|c|c|c|c|c|c|}
\hline $\begin{array}{l}\text { Pathological } \\
\text { diagnosis }\end{array}$ & Sites $\mathbf{n}$ & Normal & Abnormal & Suspicious & Normal & Abnormal & Suspicious & $\begin{array}{c}\text { Random } \\
\text { biopsy }\end{array}$ \\
\hline Normal & $46(13)$ & 29 & 16 & 1 & 9 & 34 & 3 & 7 \\
\hline Bronchitis & $125(37)$ & 72 & 50 & 3 & 41 & 70 & 14 & 29 \\
\hline Hyperplasia & $26(8)$ & 11 & 14 & 1 & 5 & 19 & 2 & 3 \\
\hline Moderate dysplasia & $52(15)$ & 22 & 29 & 1 & 3 & 39 & 10 & 2 \\
\hline Severe dysplasia & $8(2)$ & 1 & 5 & 2 & 0 & 4 & 4 & 0 \\
\hline Carcinoma in situ & $3(1)$ & 0 & 1 & 2 & 0 & 0 & 3 & 0 \\
\hline Cancer & $20(6)$ & 0 & 2 & 18 & 0 & 1 & 19 & 0 \\
\hline Total & $343(100)$ & 162 & 151 & 30 & 69 & 212 & 62 & 48 \\
\hline
\end{tabular}

Data are presented as $n$ (\%) or n. FVB: flexible video bronchoscopy; LIFE: lung imaging fluorescence endoscopy 


\begin{tabular}{|c|c|c|c|c|c|c|c|c|c|}
\hline & \multicolumn{3}{|c|}{ Normal FVB } & \multicolumn{3}{|c|}{ Abnormal FVB } & \multicolumn{3}{|c|}{ Suspicious FVB } \\
\hline \multicolumn{10}{|l|}{ LIFE } \\
\hline Normal & $2(4)$ & $46(96)$ & 48 & $1(5)$ & $20(95)$ & 21 & 0 & 0 & 0 \\
\hline Suspicious & $1(13)$ & $7(87)$ & 8 & $12(50)$ & $12(50)$ & 24 & $23(77)$ & $7(23)$ & 30 \\
\hline Total & $23(14)$ & $139(86)$ & 162 & 37 (25) & $114(75)$ & 151 & $23(77)$ & 7 (23) & 30 \\
\hline
\end{tabular}

visualised to be suspicious at LIFE, and, of these, two were suspicious and one abnormal at FVB (table 1). Of the 20 cancer lesions, 18 were suspicious and two abnormal at FVB, 19 were suspicious and one abnormal at LIFE. One cancer lesion was abnormal at both FVB and LIFE. One patient with normal LIFE and abnormal FVB had evidence of moderate dysplasia on EBX.

\section{DISCUSSION}

White light flexible fibreoptic bronchoscopy is an important diagnostic tool in the histological diagnosis of lung cancer [11]. However, it has major limitations in the diagnosis of dysplasia and early malignant lesions. Autofluorescence bronchoscopy has been reported to be useful in the diagnosis of dysplasia and early lung cancer [3, 4]. Conventionally, white light bronchoscopy is usually performed prior to autofluorescence bronchoscopy in the same session. The order in which the bronchoscopist carries out these procedures does not appear to affect the outcome of the results [7]. In this study, FVB was performed prior to LIFE. The addition of autofluorescence bronchoscopy to white light bronchoscopy has been reported to increase the detection of pre-invasive lung cancer. However, white light bronchoscopy has been commonly performed with a fibreoptic bronchoscope whilst comparing its utility with autofluorescence bronchoscopy $[3,6,7,12]$. FVB has superior optical resolution when compared with fibreoptic bronchoscopy and, therefore, has potential advantages with respect to the image resolution. To current authors' knowledge, this is the first study that has compared the utility of FVB and LIFE in the investigation of individuals at a high risk of developing lung cancer. The sensitivity of FVB in this study was $72 \%$. For FVB, the yield of abnormal and suspicious findings was significantly higher when compared with normal findings. The present study shows that EBX obtained from abnormal or suspicious areas on FVB have a good diagnostic yield in individuals at a high risk of lung cancer.

In the current study, biopsy areas that were visualised to be normal during FVB had a significant increase in yield when EBX was guided by changes noted with LIFE when compared with the random biopsies obtained when both FVB and LIFE were normal. In areas with abnormal FVB findings, there was a significant increase in the diagnostic yield of biopsies obtained from lesions that also showed abnormal and suspicious changes at LIFE when compared with normal LIFE. These findings indicate the benefit of LIFE in identifying lesions when FVB is either normal or abnormal.

An important factor associated with the success of a screening programme is the development of a diagnostic tool that is routinely available and not expensive. Following evolutions in technology, conventional white light fibreoptic bronchoscopy is now being replaced with FVB, and subepithelial vascular patterns in bronchial dysplasias have been described using a high-magnification bronchovideoscope [13]. Whether conventional FVB can be utilised for the evaluation of individuals at a high risk of lung cancer in centres that do not have the facilities for autofluorescence bronchoscopy needs to be determined. However, the good yield of FVB (72\%) in this study has led the current authors to propose that it may have a potential role in the primary evaluation of individuals at a high risk of lung cancer. The FVB used in this study had a black and white video chip, and colour on the monitor was achieved through the light source. There are no data comparing the colour video chip white light flexible bronchoscopy findings with autofluorescence bronchoscopy. Such a study would be of great interest, as the video chip white light flexible bronchoscope may have the potential to further enhance the sensitivity and specificity of FVB.

In another multicentre study, abnormal findings at white light bronchoscopy and autofluorescence bronchoscopy were considered negative [3]. In the present study, anatomical anomalies and bronchoscopic trauma were not included as abnormal findings. Furthermore, increased redness and hypervascularity, and focal thickening of a subcarina were included as abnormal findings. The sensitivity of FVB in the present study, if guided by suspicious lesions only, would be 23 out of 83 $(28 \%)$. However, abnormal lesions were used as markers for EBX, which led to a high sensitivity of FVB (72\%). Although this study does not compare the findings of fibreoptic bronchoscopy with FVB, the authors believe that the putative advantages of the FVB image assisted in the better classification of FVB findings.

The diagnostic yield of random biopsies when FVB and LIFE were normal was only $4 \%$ (two out of 48 ). When FVB was normal and LIFE showed either abnormal or suspicious changes, the diagnostic yield of biopsies guided by changes at LIFE was $18 \%$ (21 out of 114). When FVB was abnormal and LIFE normal, the yield of biopsies guided by changes at FVB 
was only $5 \%$ (one out of 21 ). Therefore, LIFE was able to distinguish FVB false-positive as well as false-negative biopsy sites. Hence, LIFE is useful both when FVB is normal or abnormal, and is also a good diagnostic tool in detecting preinvasive lesions.

In conclusion, endobronchial biopsy of suspicious findings at lung imaging fluorescence endoscopy as well as flexible video brochoscopy have a good diagnostic yield in individuals at a high risk of developing lung cancer. Lung imaging fluorescence endoscopy is more useful when flexible video bronchoscopy is either normal or abnormal.

\section{ACKNOWLEDGEMENTS}

The authors would like to thank A.C. Mehta (Cleveland Clinic Foundation, Cleveland, $\mathrm{OH}, \mathrm{USA}$ ) for reviewing the final version of the manuscript.

\section{REFERENCES}

1 Lam S, Becker HD. Future diagnostic procedures. Chest Surg Clin N Am 1996; 6: 363-380.

2 Anderson GH, Boyes DA, Benedet JL, et al. Organisation and results of the cervical cytology screening programme in British Columbia, 1955-85. BMJ (Clin Res Ed) 1988; 296: 975-978.

3 Lam S, Kennedy $\mathrm{T}$, Unger $\mathrm{M}$, et al. Localization of bronchial intraepithelial neoplastic lesions by fluorescence bronchoscopy. Chest 1998; 113: 696-702.

4 Shibuya K, Fujisawa T, Hoshino H, et al. Fluorescence bronchoscopy in the detection of preinvasive bronchial lesions in patients with sputum cytology suspicious or positive for malignancy. Lung Cancer 2001; 32: 19-25.
5 Kato H, Horai T. Color atlas of endoscopic diagnosis in early stage lung cancer. Tokyo, Wolfe publishing, 1992.

6 Venmans BJ, Linden H, Boxem TJ, Postmus PE, Smit EF, Sutedja TG. Early detection of preinvasive lesions in highrisk patients. A comparison of conventional and fluorescence bronchoscopy. J Bronchol 1998; 5: 280-283.

7 Hirsch FR, Prindiville SA, Miller YE, et al. Fluorescence versus white-light bronchoscopy for detection of preneoplastic lesions: a randomized study. J Natl Cancer Inst 2001; 93: 1385-1391.

8 The Japan Lung Cancer Society. Cytological Findings. 1st English Edn. Tokyo, Kanehara and Co., 2000; pp. 82-110.

9 Lam S, MacAulay C, le Riche J, Ikeda N, Palcic B. Early localization of bronchogenic carcinoma. Diagn Ther Endosc 1994; 1: 75-78.

10 Shibuya K, Fujisawa $\mathrm{T}$, Hoshino $\mathrm{H}$, et al. Increased telomerase activity and elevated hTERT mRNA expression during multistage carcinogenesis of squamous cell carcinoma of the lung. Cancer 2001; 92: 849-855.

11 Chhajed PN, Athavale AU, Shah AC. Clinical and pathological profile of 73 patients with lung carcinoma: is the picture changing? J Assoc Physicians India 1999; 47: 483-487.

12 Kurie JM, Lee JS, Morice RC, et al. Autofluorescence bronchoscopy in the detection of squamous metaplasia and dysplasia in current and former smokers. J Natl Cancer Inst 1998; 90: 991-995.

13 Shibuya K, Hoshino H, Chiyo M, et al. Subepithelial vascular patterns in bronchial dysplasias using a high magnification bronchovideoscope. Thorax 2002; 57: 902907. 